\title{
Healing Pattern in Calvarial Bone Defects Following Bone Regeneration in Rats Guided by Chitosan Scaffold and Adipose Tissue-Derived Mesenchymal Stem Cells
}

\author{
L. R. P. Carvalho ${ }^{1}$, N. M. Breyner ${ }^{2}$, R. C. R. Hell ${ }^{2}$, P. Valério ${ }^{2}$, S. Novikoff $^{2}$ and A. M. Goes ${ }^{* 2}$ \\ ${ }^{1}$ Department of Morphology and ${ }^{2}$ Department of Biochemistry and Immunology, Institute of Biological Sciences, \\ Universidade Federal de Minas Gerais, Belo Horizonte, Minas Gerais, Brazil
}

\begin{abstract}
Cell-based tissue engineering using scaffolds provides a promising option for the repair of bone tissue damage caused by trauma or aging-related degeneration such as osteoporosis. In this study, a porous 3D scaffold was used to support the differentiation process of rat adipose -derived stem cells (ADSCs) into osteoblasts in vitro. The scaffold was made with chitosan, gelatin and chondroitin. In addition, the scaffold was crosslinked by glutaraldehyide. The osteogenic differentiation of ADSCs was improved in 3D culture as shown by the cell viability assay (MTT) and analyses of, alkaline phosphatase activity (ALP), and collagen production during three weeks of culturing. During the 2nd and 3rd weeks of culturing, bone markers, such as osteopontin and osteocalcin, were detected by the PCR analysis. In vivo biocompatibility was evaluated subcutaneously in rats. A mild inflammatory response was observed during the 5 weeks. Reduction of the matrix fibers by reabsorptive cells and formation of new blood vessels were observed during this period. However, no inflammation was observed. Five weeks after the implants were placed in the calvaria -defects, a small amount of bone repair was observed. In addition, immunohistochemistry revealed the presence of EGFPs ADSCs in the newly formed extracellular matrix. These findings indicated that the chitosan-gelatin-chondroitin 3D structure enhances cellular proliferation, which may be useful in the development of biomaterials for the stimulation of adult stem cells in bone tissue engineering.
\end{abstract}

Keywords: Bone healing, calvaria repair, chitosan-gelatin-chondroitin scaffold, EGFP-transgenic Lewis rats, mesenchymal stem cells and tissue engineering.

\section{INTRODUCTION}

Bone is type of tissues that is capable of both remodeling and regeneration. It is a remarkable organ that has key roles in the following critical functions of human physiology: protection, movement and support of other critical organs; blood production; and blood $\mathrm{pH}$ regulation and others [1]. The importance of bone becomes clear in the case of diseases, such as osteoporosis and osteomyelitis, in addition to traumatic injury in which bone does not function properly [2-4]. The clinical and economical impacts of bone defect treatments are staggering [5-8]. Autografts remain the clinical gold standard for bone repair. Unfortunately, donor site morbidity, cosmetic concerns, and prolonged hospitalization are just a few of the drawbacks of this procedure $[9,10]$. Similarly, the use of allograft presents risks due to immune rejection, inappropriate biodegradation, and disease transmission [11]. Recently, tissue engineering has emerged as a new method involving the combination of cells, scaffolds, and bioactive agents to fabricate functional new tissues to replace damaged tissues. The cells used to repair or regenerate tissues and organs can be recruited from the receiver, or they can be exogenously provided. Adipose tissuederived mesenchymal stem cells (ADSCs) share many cha-

*Address correspondence to this author at the Department of Biochemistry and Immunology, Institute of Biological Sciences, Universidade Federal de Minas Gerais, Av. Antônio Carlos, 6627 ICB - UFMG, 31270-910 - Belo Horizonte, MG, Brazil; Tel: +55 31 34092639; Fax: +55 31 34092810; E-mail: goes@icb.ufmg.br racteristics of bone marrow stem cells (BMSCs), including the potential for extensive proliferation and multilineage differentiation. Moreover, compared to BMSCs, adipose tissue provides additional benefits, such as a source of stem cells. ADSCs can be isolated via minimally invasive procedures from the subcutaneous fat of patients. ADSCs are highly expandable in culture, and they can be readily induced to differentiate into bone tissue-forming cells by exposure to osteogenic medium [12-14]. Thus, ADSCs emerge as great candidates for tissue engineering applications. The need for bone repair has led to the development of bone grafts using tissue- engineering approaches. Bone tissue engineering has been one of the most promising areas of research, and it has provided a potential clinical application to cure bone defects. Thus, there is a need to identify biomaterials to meet the challenging combination of biological, mechanical, and degradative features required for bone tissue engineering [15-17]. Recently, chitosan (CS) has garnered much attention for its use in bone tissue engineering [18-22]. For improving the mechanical and biological properties of chitosan over a broad range, blending chitosan with other polymers has been investigated [21]. Gelatin is blended with chitosan to improve its biological activity because it contains an Arg-Gly-Asp-like sequence that promotes cell adhesion and migration [20, 23]. Moreover, inclusion of chondroitin sulfate in the scaffold may promote phosphatase alkaline production and mineralization in vitro. Chitosan-gelatin scaffolds haves been produced with and without the use of crosslinkers, such as glutaraldehyde [24]. 
In this study, a 3D tri-copolymer scaffold was produced from chitosan, gelatin and chondroitin sulfate to mimic bone matrix and to support ADSCs osteogenic differentiation. Secretion capability and mineralization characteristics were analyzed to verify the osteogenic process and biomaterial osteoinduction properties.

\section{MATERIALS AND METHODOLOGY}

\section{Scaffold Synthesis of a 3D Chitosan-Gelatin-Chondroitin Scaffold}

A $0.7 \%(\mathrm{w} / \mathrm{v})$ chitosan (Sigma, USA) solution, $0.7 \%$ $(\mathrm{w} / \mathrm{v})$ gelatin (Vetec) solution and $0.1 \%(\mathrm{w} / \mathrm{v})$ chondroitin (Sigma, USA) solution were prepared and diluted in $1 \mathrm{M}$ acetic acid at room temperature. . These three solutions were then mixed in a ratio of 3:1:0.5 (v/v) and crosslinked with $0.1 \%(\mathrm{v} / \mathrm{v})$ glutaraldehyde (Sigma, USA) at $25 \%$. The final solution was dispensed in a volume of $1 \mathrm{ml} / \mathrm{well}$ in a 24 -well culture plate (Costar). The plates were frozen at $-20^{\circ} \mathrm{C}$ for 12 hours, and the samples were then lyophilized for 16 hours. Then, the scaffolds were treated with received once again 0.1 $\%(\mathrm{v} / \mathrm{v})$ glutaraldehyde, and they were frozen and lyophilized again. All scaffolds were sterilized with $\gamma$ irradiation from a Co-60 source at $15 \mathrm{kGy}$.

\section{Scanning Electron Microscopy}

Scanning electron microscopy (SEM) was used to characterize the morphology and structure of the scaffold. The specimens in 3D culture were fixed in a solution containing $2.5 \%$ glutaraldehyde and $0.5 \%$ paraformaldehyde in phosphate buffer $(\mathrm{pH}$ 7.4). The samples were then trimmed, osmium treated and dehydrated. The scaffolds were coated with gold (Sputter Coater; SPI Supplies) for $90 \mathrm{~s}$ at $13 \mathrm{~mA}$. The images were taken in the Department of Metallurgical and Materials Engineering at the Federal University of Minas Gerais in Brazil using a scanning electronic microscope (JEOL6360LV) at $15 \mathrm{kV}$ and $750 \mathrm{~mA}$.

\section{ADSCs Isolation and 2D and 3D Cultures}

Adipose-derived stem cells were obtained from 4-weekold Lewis rats purchased from Centro de Bioterismo ICB, UFMG (Belo Horizonte, Brazil) or from Lewis transgenic rats carrying the enhanced green fluorescent protein (EGFP) genes (LEW-Tg EGFP F455/Rrrc; Rat Resource and Research Center, University of Missouri, USA), which were used to obtain endogenously EGFP-labeled cells. All experiments in this work were submitted and approved by the Ethics Committee of Federal University of Minas Gerais under the protocol number 154/2006. The rats were anesthetized with a combination of ketamine $(60 \mathrm{mg} / \mathrm{kg}$; Dopalen AgriBrands Ltd.) and xylazine (10 mg/kg; AgriBrands Ltd.). The inguinal fat pads were harvested and extensively washed with phosphate-buffered saline (PBS, Gibco, USA). They were then excised, finely minced, and rinsed three times in phosphate-buffered saline for 5 minutes followed by digestion with $0.15 \%$ collagenase (Gibco, USA) and vigorous shaking for 90 minutes at $37^{\circ} \mathrm{C}$ in a 50 -cc centrifuge tube. Next, an equal volume of Dulbecco's modified Eagle's medium (DMEM, Gibco, USA) containing 10\% fetal bovine serum (FBS; Sorali Biotecnologia, Brazil) was added to neutralize the collagenase. The cell suspension was then centrifuged at $1400 \mathrm{rpm}$ for 10 minutes, and the cell pellet was resuspended with control medium. Finally, the cells were cultured in $75 \mathrm{~cm}^{2}$ - tissue culture flasks (TPP-Techno Plastic Products, Trasadingen, Switzerland) containing control medium (CM) consisting of Dulbecco's modified Eagle's medium containing $10 \%$ fetal bovine serum, $100 \mathrm{U}$ ml-1 penicillin, $100 \mu \mathrm{g} \mathrm{ml}-1$ streptomycin, and $0.25 \mu \mathrm{g} \mathrm{l-1}$ Fungizone (Gibco, USA) at $37^{\circ} \mathrm{C}$ in a humidified atmosphere of $5 \% \mathrm{CO}_{2}$ and $95 \%$ air. The nonadherent cells were removed by washing the cultures with medium exchanges every 2 days. The control medium was changed 2 or 3 times a week, and the cells were expanded in control medium. The adherent cells were allowed to grow to approximately $90 \%$ confluency, and subsequently, they were detached with a $0.05 \%$ trypsin/EDTA solution and split at a 1:3 ratio. At the fourth passage, the cells were released by trypsinization, and they were then centrifuged and resuspended. ADSCs seeded on the scaffold were defined as 3D cell cultures. For positive controls of the in vitro experiments, ADSCs were plated in wells without scaffolds, which were characteristic of a monolayer culture, and these cells were considered a two dimensional cell culture (2D) cell culture. In both cases, ADSCs $\left(1 \times 10^{6} / \mathrm{mL} /\right.$ well $)$ were expanded in control medium until the fourth passage, and they were then plated in 24-well culture plates (Nunc) for 2D (monolayer culture) and 3D culture evaluation. In this phase of the study, the following two types of culture mediums were used: control medium and osteogenic medium.

\section{Osteogenic Differentiation of ADSCs in 2D and 3D Culture}

After primary culture in the control medium, the cells were trypsinized and replated into 24-well tissue culture plates at a density of $1 \times 10^{6}$ cells per well in monolayer culture. The same number of cells $\left(1 \times 10^{6}\right)$ was seeded per scaffold in 3D culture. The cells were incubated in the control medium for 24 hours to allow attachment, and the medium was then changed to osteogenic medium (OS). OS medium is composed of the following components: Dulbecco's modified Eagle's medium containing 10\% fetal bovine serum, $10^{-8} \mathrm{M}$ dexamethasone (Sigma, USA), $10 \mu \mathrm{M}$ $\beta$-glycerophosphate (Merck, Darmstadt, Germany) and 50 $\mu \mathrm{g} / \mathrm{ml}$ ascorbic acid (Sigma, USA). The medium was changed once every 3 days.

\section{Flow-Cytometric Analysis}

After the fourth passage, ADSCs were released by trypsinization. The cells (Approximately $1 \times 10^{6}$ cells/well) were then incubated with a set of mouse anti-rat CD45, CD90, CD73 and CD54 antibodies (BD Pharmingen, USA) diluted 1: 50 for $30 \mathrm{~min}$ at $4^{\circ} \mathrm{C}$. After washing with PBS, the cells were incubated with an Alexa 488-conjugated antimouse antibody (Sigma, USA) for $30 \mathrm{~min}$ at $4^{\circ} \mathrm{C}$, and the cells were then washed with PBS. The cells were fixed with $200 \mu \mathrm{l}$ of $2 \%$ formaldehyde, and they were analyzed by flow cytometry using a FACSCalibur (Becton Dickinson, USA). The data were analyzed with CellQuest Software (BD Biosciences, USA). Approximately 30,000 events were acquired. 


\section{Cell Viability Assay}

The viability of the cells in $2 \mathrm{D}$ and $3 \mathrm{D}$ culture was evaluated weekly using a 3-(4,5-dimethylthiazol-2-yl)-2,5diphenyl tetrazolium bromide (MTT) assay. In 2D culture, the cells $\left(1 \times 10^{6}\right.$ cells/well $)$ were seeded into 24 -well plates. In $3 \mathrm{D}$ culture, $1 \times 10^{6}$ cells were seeded into each scaffold. The scaffolds were placed in 24-well plates. Briefly, MTT (5 $\mathrm{mg} / \mathrm{ml}$ ) was added to each well of 2D and 3D cultures, and the cells were then incubated in a humidified incubator with $5 \% \mathrm{CO}_{2}$ at $37^{\circ} \mathrm{C}$. Two hours later, cell morphology and formazan salts were visualized using an inverted optical microscopy. The formazan salts were dissolved with $10 \%$ SDS in $\mathrm{HCl}$ overnight, and the optical density measurement was performed at $595 \mathrm{~nm}$. For both the monolayer and matrix samples, control medium was used the first day of culture (24 hours) and osteogenic medium was used for the remaining culture times (1,2 and 3 weeks). The MTT assay was performed on cells from each culture period. The values of all samples were taken relative to the value of the control, and the values were expressed as percentages. The controls consisted of unstimulated ADSCs in a monolayer or 3D culture for 24 hours, and an activity of $100 \%$ was attributed to the controls. The percentages of the other groups were defined by the following formula: cellular viability $(\%)=$ $100 \times(\mathrm{S} / \mathrm{C})$ where $\mathrm{S}$ is the absorbance value of the cells treated with osteogenic media and $\mathrm{C}$ is the absorbance value of the cells in control media (2D or 3D). Triplicate samples were obtained in three separate experiments.

\section{Osteogenic Pathway of ADSCs in Monolayer and 3D Scaffold Cultures}

Osteogenesis was confirmed by means of von Kossa staining (to highlight calcification of extracellular matrix; ECM), assessment of alkaline phosphatase (ALP) activity and measurement of collagen production at 24 hours and after 1,2 and 3 weeks of initial osteogenic induction. Osteogenesis was also confirmed by the expression of osteopontin and osteocalcin genes.

\section{Alkaline Phosphatase Activity}

Cell alkaline phosphatase (ALP) activity was measured by a (5-bromo, 4-chlorine, 3-indolylphosphate-nitroblue tetrazolium (BCIP-NBT) assay (Gibco, USA). Briefly, 300 $\mu l$ of the supernatant from each well was removed and mixed with $100 \mu 1$ of BCIP-NBT solution. After 2 hours of incubation, the optical density measurement was performed at $595 \mathrm{~nm}$ using an ELISA reader. For both the monolayer and matrix samples, control medium was use for the first day of culture (24 hours) and osteogenic medium was used for the remaining culture times (1, 2 and 3 weeks). The BCIPNBT assay was performed on cells from each culture period. The values of all samples were taken relative to the value of the control, and the values were expressed as percentages. The controls consisted of unstimulated ADSCs in monolayer or $3 \mathrm{D}$ culture for 24 hours, and an activity of $100 \%$ was attributed to the controls. The percentages of the other groups were defined by the following formula: cellular viability $(\%)=100 \times(\mathrm{S} / \mathrm{C})$, where $\mathrm{S}$ is the absorbance value of the cells treated with osteogenic media and $\mathrm{C}$ is the absorbance value of the cells in control media (2D or $3 \mathrm{D})$.
Triplicate samples were obtained in three separate experiments.

\section{Collagen Production}

Collagen production levels were measured using the SIRCOL Collagen Assay (Biocolor, Ireland). Collagen present in the supernatant was precipitated by the stain. The collagen was then dissolved, and the absorbance at $595 \mathrm{~nm}$ was read using an ELISA reader. For both the monolayer and matrix samples, control medium was used for the first day of culture (24 hours) and osteogenic medium was used for the remaining culture times (1,2 and 3 weeks). The values of all samples were taken relative to the value of the control, and the values were expressed as percentages. The controls consisted of unstimulated ADSCs in monolayer or $3 \mathrm{D}$ culture for $24 \mathrm{~h}$. The percentages of the other groups were defined by the following formula: cellular viability (\%) $=100 \times(\mathrm{S} / \mathrm{C})$, where $\mathrm{S}$ is the absorbance value of the cells treated with osteogenic media and $\mathrm{C}$ is the absorbance value of the cells in control media (2D or 3D). Triplicate samples were obtained in three separate experiments.

\section{Reverse Transcriptase-PCR}

In $2 \mathrm{D}$ and $3 \mathrm{D}$ cultures of ADSCs, the relative mRNA levels of osteopontin, osteocalcin and $\beta$-actin were determined before and after 24 hours and 3 weeks of osteogenic differentiation. The samples were mechanically homogenized, and total RNA was extracted using TRIzol reagent according to the manufacturer's protocol (Invitrogen Life Technologies, USA). RNA $(1 \mu \mathrm{g})$ was reverse transcribed in the presence of $1 \mathrm{mM}$ dNTPs, $1 \mathrm{x}$ reverse transcriptase (RT) buffer, $200 \mathrm{U}$ M-MuLV RT, 20 U ribonuclease inhibitor and 40 pmol oligo-dT primer with a final reaction volume of 20 $\mu 1$. The reactions were incubated at $42^{\circ} \mathrm{C}$ for 60 minutes, and the RT was inactivated at $70^{\circ} \mathrm{C}$ for 10 minutes. PCR was performed after an initial denaturation for 10 minutes at $95^{\circ} \mathrm{C}$, followed by 25 cycles of amplification $\left(9^{\circ} \mathrm{C}\right.$ for 15 segunds followed by $60^{\circ} \mathrm{C}$ for 1 minute). The PCR samples contained 5 pmol of each forward and reverse primer in addition to $4 \mu \mathrm{l}$ of cDNA template with a final volume of $20 \mu 1$. All reactions were normalized to the $\beta$-actin housekeeping gene. A 'no template' control containing nongenetic material was included to control for contamination or nonspecific reactions. Each sample was tested in duplicate. The following forward $(\mathrm{F})$ and reverse $(\mathrm{R})$ primers were used: osteopontin, TCCCAGTTGCCCAGCGTCTGA (F) and GCTGGCTTTGGAACTCGCCTGA (R); osteocalcin, AGCCTTCATGTCCAAGCAGGAG (F) and TAAACGGT GGTGCCATAGATGC (R); and $\beta$-actin, CTCCTTAATGT CACGCACGATTTC (F) and GTGGGGCGCCCCAGGC ACCA (R).

\section{Von Kossa Staining}

Cells plated in 6-well plates were rinsed with phosphatebuffered saline and fixed in $4 \%$ paraformaldehyde for 1 hour. The cells were then incubated in 5\% silver nitrate for 30 minutes in the dark. Following incubation, the cells were rinsed with distilled water and exposed to ultraviolet light for 1 hour. Secretion of calcified extracellular matrix was confirmed as black nodules with von Kossa staining. 


\section{Surgical Procedure}

\section{Subcutaneous Implants}

Sixteen Lewis rats were used for subcutaneous implantation of the chitosan- scaffolds. Each animal was anaesthetized of ketamine combined with xylazine hydrochloride (12 $\mathrm{mg} / 100 \mathrm{~g}$ body weight) and subjected to local tricotomy for adequate surgical procedure. The skin was disinfected and under surgical sterile conditions, and incisions of approximately $2 \mathrm{~cm}$ length were performed through the cutis and subcutis. After cutaneous incising the scaffolds were inserted and the skin carefully sutured. At 1, 3 and 5 weeks postimplantation, the animals the rats were euthanized using $\mathrm{CO}_{2}$ inhalation. The scaffolds and surrounding tissue were explanted for further evaluation.

\section{Critical-Sized Calvarial Defects in Rats}

The critical-sized calvarial defects were prepared according to previously described methods [25]. All experiments in this work were submitted and approved by the Ethics Committee of Federal University of Minas Gerais under the protocol number 154/2006. Adult Lewis rats were used to test the enhancing effect of ADSCs-based bone regeneration for repairing calvarial defects. The cell/scaffold constructs were implanted into rats under anesthesia by intraperitoneal injection (10 mg/100 $\mathrm{g}$ body weight) of ketamine combined with xylazine hydrochloride $(12 \mathrm{mg} / 100$ $\mathrm{g}$ body weight). The hair over the calvarium was shaved and cleaned with a depilator. A midline calvarial incision was made, and a 7-mm hole was drilled penetrating through the calvarial bone using a trephine burr with constant PBS irrigation. Extreme care was taken to avoid damaging the dura mater. Twenty-four rats with calvarial defects were randomly allocated to 3 groups (08 rats/group) according to the following cell/scaffold implants and treatments: rats with critical-sized (7-mm) calvarial defects (control); rats with critical-sized (7-mm) calvarial defects were implanted with chitosan only or ADSCs-seeded chitosan in the defect sites. The wounds were sutured after implantation with nylon $5 \mathrm{e} 0$ sutures. All rats survived and had no wound infections throughout the experiment. At 30 days post-implantation, the rats were euthanized using $\mathrm{CO}_{2}$ inhalation. Calvarial specimens were harvested, fixed in $4 \%$ paraformaldehyde at $4^{\circ} \mathrm{C}$ for 24 hours and evaluated histologically. At five weeks post-implantation, calvarial specimens were harvested and evaluated histomorphometrically. All calvarial specimens were decalcified, fixed and paraffin-embedded as previously described [25]. The 5-mm thick bone sections were removed and stained with hematoxylin and eosin (H\&E) (Santa Cruz, Santa Cruz, CA, USA). A microscope with 10x magnification equipped with a digital CCD camera (Eclipse 50i; Nikon Inc., Michigan, USA) was used to acquire images of the sections.

\section{Cell Tracing with Immunohistochemistry Analysis}

To track the fate, distribution and differentiation of the transplanted ADSCs after delivery on the scaffold, the presence of EGFP was used as a tag. The slices were deparaffinized in xylene, rehydrated through a graded series of ethanol and washed in PBS. The sections were then immersed in $3 \% \mathrm{H}_{2} \mathrm{O}_{2}$ (1 hour) to neutralize the endogenous peroxidase activity followed by incubation with $2 \%$ BSA in
PBS (1 hour) for the blocking of nonspecific binding sites. Afterward, the sections were incubated overnight at $4^{\circ} \mathrm{C}$ with rabbit anti-GFP (1:100, Abcam, Cambridge, UK). The secondary biotinylated anti-rabbit antibody (Universal $\mathrm{LSAB}^{\mathrm{TM}}+\mathrm{Kit} / \mathrm{HRP}, \mathrm{Rb} / \mathrm{Mo} /$ Goat K0690-1Dako, Glostrup, Denmark) was applied for 30 minutes at room temperature. After washes with PBS, the sections were incubated with a streptavidin-horseradish peroxidase conjugate for 30 minutes at room temperature. The reaction was localized with a solution containing $350 \mu \mathrm{M} 3,3^{\prime}$-diaminobenzidine (Sigma Chemical Co.) and $1 \% \mathrm{H}_{2} \mathrm{O}_{2}$ in PBS. The slides were counterstained with Mayer's hematoxylin. All samples were processed at the same time to avoid differences among the assays. Negative controls were obtained by the omission of the primary antibody.

\section{Statistical Analysis}

All experiments were repeated three or more times with triplicate samples, and the data were represented as the means +/- standard derivations. Statistical analysis was performed by one-way analysis of variance (ANOVA) and Bonferroni's post-hoc test (GraphPadSoftware, San Diego, Calif., USA). Differences were considered statically significant as follows: $* p<0.05, * * p<0.01$ and $* * * p<0.001$.

\section{RESULTS AND DISCUSSION}

In this study, a 3D chitosan-gelatin-chondroitin scaffold cross-linked by glutaraldehyde was used to induce ADSCs differentiation into an osteoblast lineage in vitro. Moreover, the biocompatibility and biodegradability of the scaffold in addition to bone regeneration in the subcutaneous implant and critical-sized calvarial defects in rats were evaluated using a bone defect model in vivo. Flow cytometry analysis demonstrated that isolated ADSCs expressed CD45 (3\%), and the analysis demonstrated that a significant percentage of these ADSCs were positive for CD54 (95\%), CD73 (94\%) and CD90 (87\%) (data not shown). The high expression of the non-hematopoietic markers, CD54, CD73 and CD90, confirmed the purity level of the mesenchymal stem cells (MSCs). These results were in agreement with previous studies [26, 27]. The isolated cell population exhibited a specific surface antigen expression pattern that is characteristic of mesenchymal stem cells as previously reported $[23,28]$. The ADSCs rapidly proliferated displayed a fibroblastic morphology adhered to plastic (Fig. 1a). However, when the ADSCs were cultured in the presence of osteogenic media, changes in cell morphology were observed. After the seventh day of culture in osteogenic media, cells assumed a cubic shape similar to typical osteoblast morphology (Fig. 1b), and they began to cluster in structures called bone nodules (Fig. 1c). The ability of ADSCs to differentiate into osteoblast cells has been previously reported [21, 29]. In this study, a specific medium was used to induce differentiation. The MTT, alkaline phosphatase and collagen production assays were performed to assess if the cells were viable and proliferating in the presence of osteoblast differentiation medium. After addition of the osteogenic culture medium, a slight change was observed in the morphology of the cells after incubation for 1 and 2 weeks. After 3 weeks of osteogenic induction, the fusiform morphology was replaced by a round shape. When osteo- 


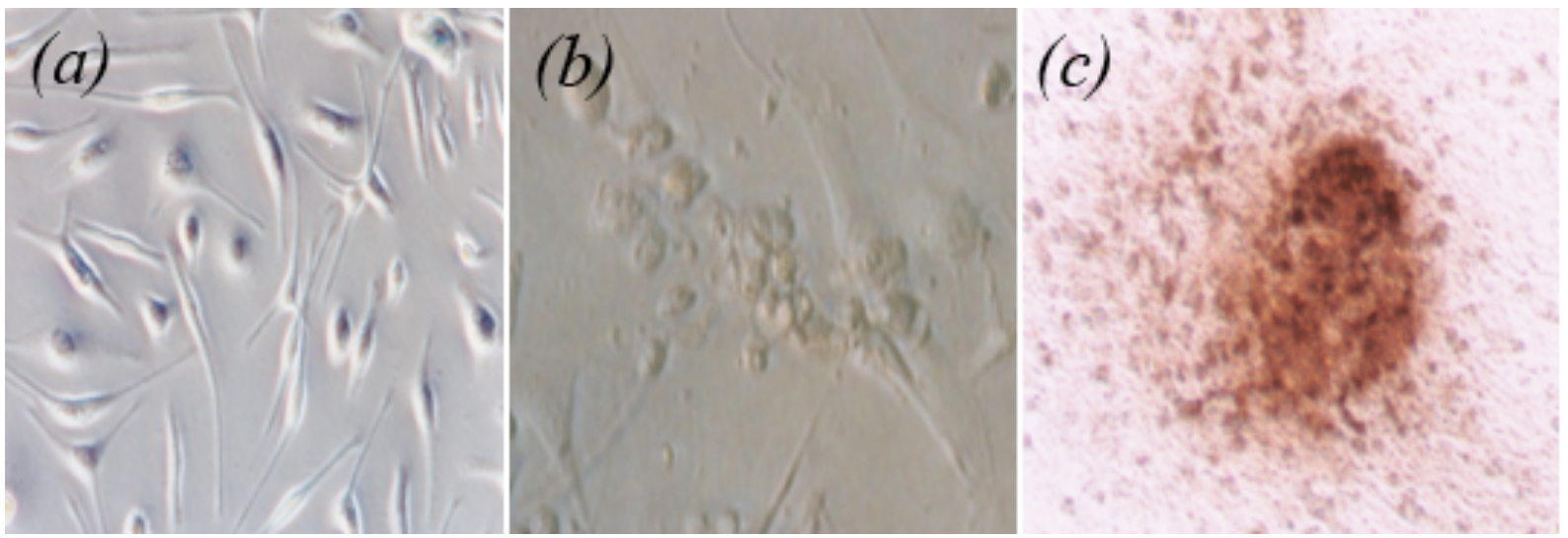

Fig. (1). ADSCs in monolayer culture. a) After 10 days of culture, the ADSCs had fibroblast-like aspects. Magnification of 200x. b) After 7 days of culture in osteogenic medium, the cells displayed a round morphology similar to osteoblasts. Magnification of 300x. c) After 21 days of culture in osteogenic medium, the cells began to cluster in structures called bone nodules. Magnification of 40x.

blasts morphologically change and present a round shape $[23,30]$, it is characteristic of osteogenesis [31]. Osteogenic induction of human adipose stem cells [32], mouse adipose stem cells [33] and rat adipose cuboidal shape, forming several bone nodules by the second week of osteogenic induction, and forming many nodules by the fourth week of the induction.

In this study, a 3D chitosan-gelatin-chondroitin scaffold cross-linked by glutaraldehyde was constructed to induce ADSCs differentiation into an osteoblast lineage in vitro with a purpose to promote in vivo bone healing in calvaria defects. The synthesized chitosan-gelatin-chondroitin scaffold had a high porosity with interconnected pores; in addition, the pore sizes were random and larger than $300 \mu \mathrm{m}$ (Fig. 2a). These observations were in agreement with a previous report by Machado et al. [21]. This highly porous structure allows cell penetration, growth, differentiation and proliferation. After 3 days in 3D culture, the ADSCs were spread out on the scaffold, and they established close contact with the biomaterial (Fig. 2b, c). This highly porous structure allows cell penetration, growth, differentiation and proliferation. After 3 days in 3D culture, the ADSCs were spread out on the scaffold, and they established close contact with the biomaterial (Fig. 2b, c). Chitosan is one of the most promising biopolymers for tissue engineering, and it can potentially be used for bone regeneration applications [19, $20,23,34,35]$. Gelatin and chondroitin have been employed to improve cell adhesion and activation in 3D cultures [20, 34, 36, 37]. Moreover, sponge-type scaffolds have been produced by a freeze-drying method, which is a gentle drying method to obtain porous scaffolds with interconnecting pores $[21,24,26]$. In addition, chitosan-gelatin scaffolds have been formed with cross-linkers, such as glutaraldehyde [23]. When cultured, the ADSCs were viable, and they demonstrated a capacity to convert MTT into formazan crystals after 24 hours and after 1, 2 and 3 weeks in 2D (Fig. 3a) and 3D cultures (Fig. 3b). In ADSCs 2D culture, the rate formation of formazan crystals remained constant until the second week of osteogenic induction, but the rate then significantly decreased $(p<0.0001)$ by approximately $40 \%$ in the third week of culture compared to the control (Fig. 3a, g). When ADSC cultivation was supported by the 3D scaffold, there was a significant increase $(p<0.0001)$ in MTT rates after the second week of cultivation in osteogenic medium, and the increase corresponded to $20 \%$ of the absorbance measured in the control (Fig. 3b). However, after the $3^{\text {rd }}$ week, there was a significant reduction $(p<0.005)$ in levels of MTT with the decrease corresponding to $10 \%$ of the absorbance measured in the control. When the cells mature and become differentiated, it is common that some of them die [34, 38]. These data, suggest that the chitosan-gelatin-chondroitin scaffolds had low cytotoxicity. These results demonstrate that chitosan associated with gelatin and chondroitin enhances the process of osteogenesis and maintains the viability of cells while the
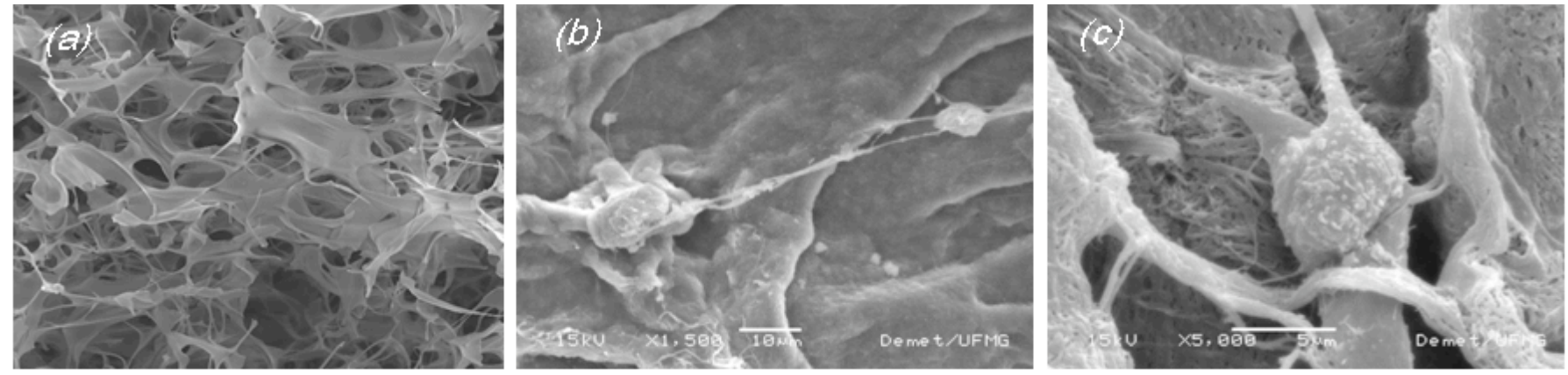

Fig. (2). Cross-sectional SEM images of the chitosan-gelatin-chondroitin scaffold. a) SEM photomicrographs showing a porous structure with interconnectivity between the pores. Magnification of 200x. b) ADSCs in chitosan-gelatin-chondroitin scaffold after 72 hours. The adhesion and interconnection of cells in the scaffolds are shown. Magnification of 300x. c) A cells attached to the pore after 72 hours. Magnification of 300x. 
differentiation process occurs. In addition, we investigated ALP activity and collagen production to analyze possible phenotypic alterations in monolayer or 3D scaffold cultures. The ALP activity was detected in 2D and 3D cultures under
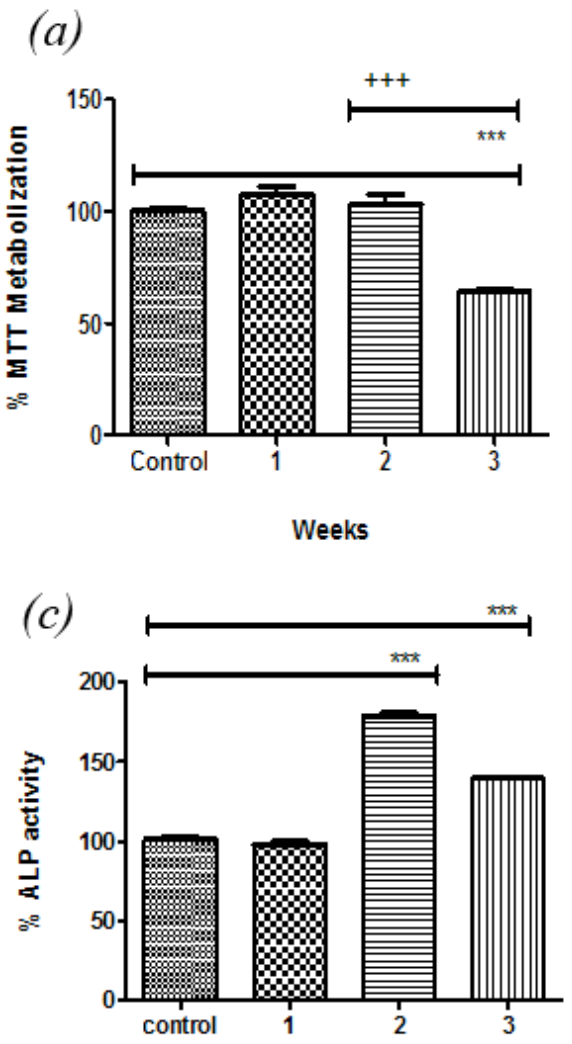

(e)

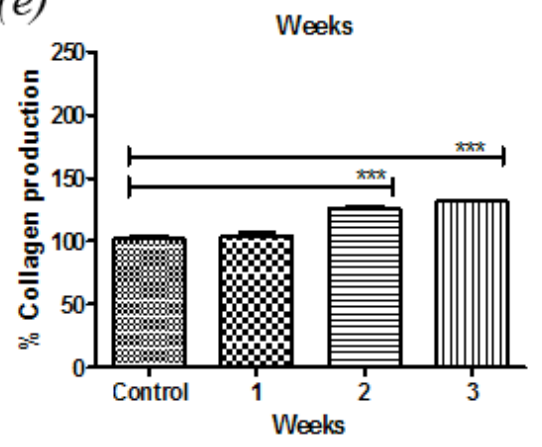

both culture mediums for all the evaluated time points. The ALP activity in monolayer cultures significantly increased ( $p$ $<0.0001$ ) after the second week of osteogenic induction, and it reached levels $80 \%$ higher than the levels found in the
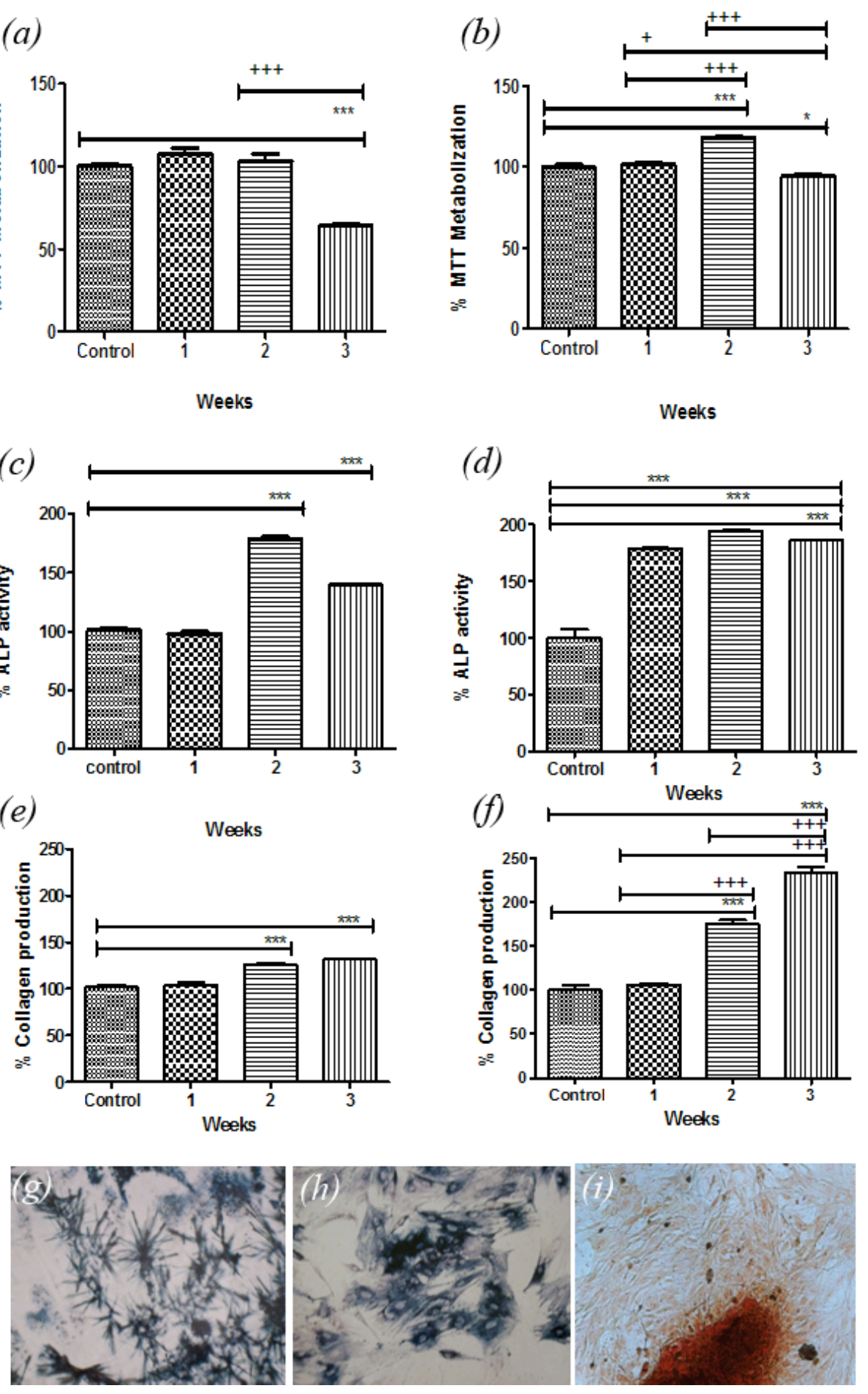

Fig. (3). ADSC activity during osteogenic differentiation in $2 \mathrm{D}$ and $3 \mathrm{D}$ cultures in a chitosan-gelatin-chondroitin scaffold. ADSCs were cultured in osteogenic medium for 3 weeks. a) Cell viability (MTT) in 2D culture. b) Cell viability (MTT) in 3D culture. c) ALP activity in 2D culture. d) ALP activity in 2D culture. e) Collagen production in 2D culture. f) Collagen production in 3D culture. g) Visualization of formazan crystals by optical microscopy in the MTT assay. The spreading of formazan crystals was observed indicating the presence of viable cells. Magnification of 150x. h) Photographic representation of ALP activity. Purple precipitate indicates osteogenesis of ADSCs. i) Photographic representation of collagen production. Magnification of 150x. The results are presented as the mean $+/-$ SD of triplicates from three experiments. The symbols represent the following $p$-values: ${ }^{*} p<0.05$ compared to controls; $+p<0.05$ compared between groups; ${ }^{* * *} p$ $<0.0001$ compared to controls; and ${ }^{+++} p<0.0001$ compared between groups. 
control. In the third week of culture, however, there was a decrease $(p<0.0001)$ in ALP activity when compared to the second week. Despite the decrease in ALP activity in the third week of cultivation, there was still significant ALP activity $(p<0.0001)$ compared to control cells (Fig. 3c, h). When ADSCs were cultivated in the 3D scaffold, significant increases $(p<0.0001)$ in the alkaline phosphatase activity were observed from the first week to the end of cultivation in osteogenic medium. Compared to the control cultures, the increases in alkaline phosphatase activity were 80,95 and $80 \%$ for the $1^{\text {st }}, 2^{\text {nd }}$ and $3^{\text {rd }}$ week of cultivation, respectively (Fig. 3d). These observations were in accordance with early differentiation of these cells into immature osteoblasts, which is characterized by ALP enzyme activity [39]. Bone ALP is produced in extremely high amounts during the bone formation cycle [40]. Although ALP has been detected in stem cell cultures [41], the level of bone ALP is higher than the level of stem cell ALP, which is considered the basal level. Moreover, the secretion of total collagen by the monolayer and 3D cultured ADSCs in osteogenic medium is an indication of a differentiated cell phenotype. These results demonstrated that the production of collagen reaches levels approximately $30 \%$ higher $(p<0.0001)$ than the levels reached with the control when cultured in a monolayer from the second week of cultivation with the increase maintained in the $3^{\text {rd }}$ week (Fig. 3e, i). With regard to the 3D cultures, cells cultured in osteogenic medium showed an early and significant increase $(p<0.0001)$ in collagen production. In the $2^{\text {nd }}$ week of osteogenic induction, collagen production rates increased by $60 \%$ compared to the control, and collagen production rates in week 3 increased by $130 \%$ compared to the control (Fig. 3f).

When considering that mineralization is a process that occurs during osteogenic differentiation in vitro [42], we decided to analyze the influence of chitosan associated with gelatin and chondroitin in mineralized structure formation. When counting the nodules of mineralization, the number of nodules in $2 \mathrm{D}$ culture was lower than in $3 \mathrm{D}$ culture. In monolayer cultures, the number of nodules was approximately 2, 6 and 8 nodes per field after 1,2 and 3 weeks of osteogenic induction, respectively (Fig. 4a, c). In 3D cultures of ADSCs, the quantification of bone nodules was 7, 16 and 26 nodules per field after 1,2 and 3 weeks of osteogenic induction, respectively (Fig. $\mathbf{4 b}$, d). These results demonstrated that differentiation of ADSCs to osteoblasts promoted the increase of mineral nodules in the presence of the $3 \mathrm{D}$ matrix.

Another important aspect to be evaluated in the differentiation of ADSCs to osteoblasts is the gene expression of osteopontin [21, 43] and osteocalcin [21, 44]. After 21 days of differentiation, the expression of both osteopontin and osteocalcin was detected in both 2D and 3D cultures (Fig. 5) with osteocalcin expressed at a lower level than osteopontin.
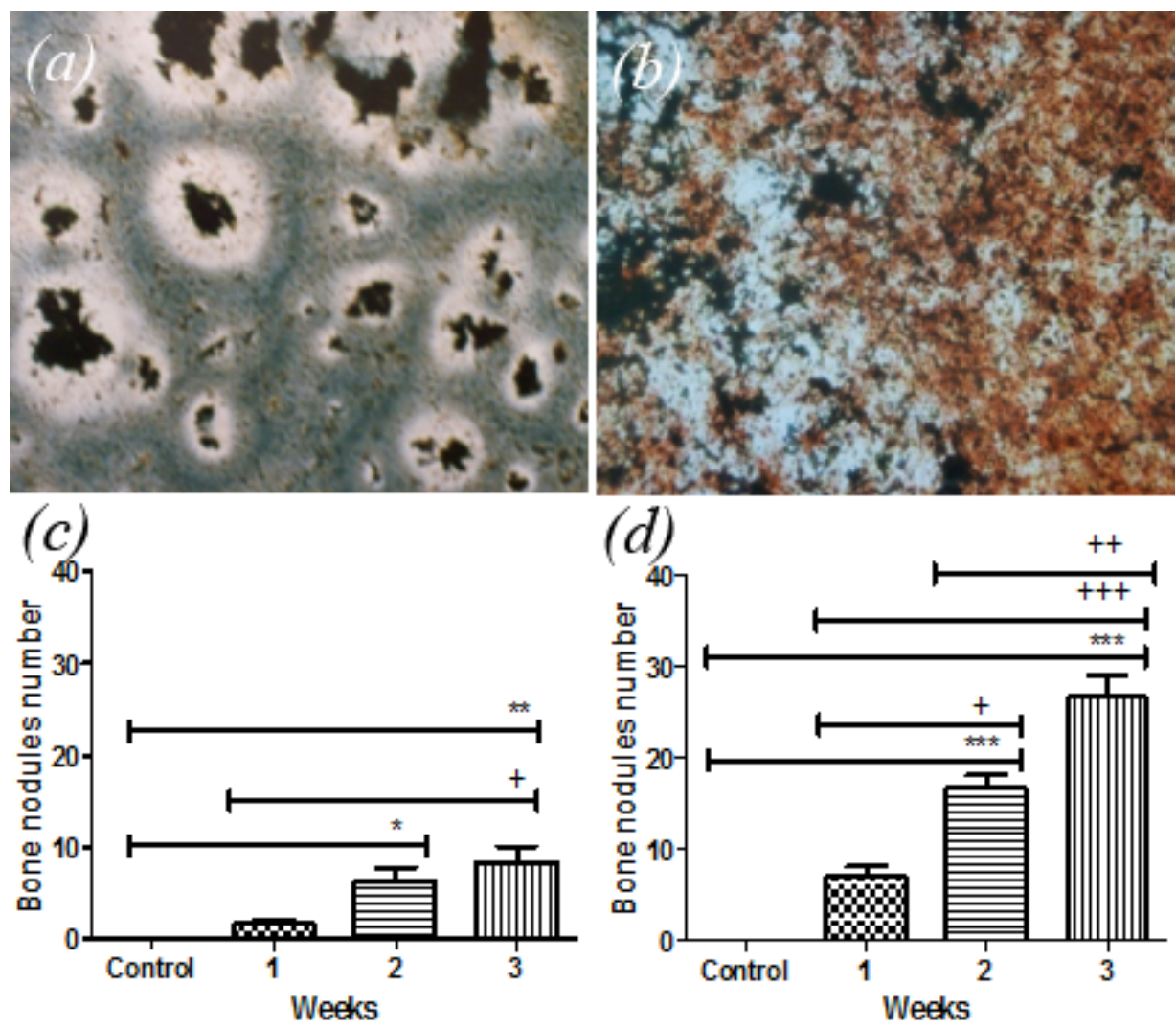

Fig. (4). Von Kossa staining. Osteogenic differentiation was also assessed by von Kossa staining. Secretion of a calcified extracellular matrix was observed as black nodules. ADSCs were cultured in osteogenic medium for 3 weeks. a) Von Kossa staining in ADSCs in 2D culture. b) Von Kossa staining in ADSCs in 3D chitosan-gelatin-chondroitin matrices. c) Quantification of mineralized structures in 2D culture. d) Quantification of mineralized structures in 3D culture. The results are presented as the mean $+/-$ SD of triplicates from three experiments. The symbols represent the following $p$-values: ${ }^{*} p<0.05$ compared to controls; $+p<0.05$ compared between groups; ${ }^{* *} p<0.002$ compared to controls; and ${ }^{++} p<0.002$ compared between groups. 
Thus, these results demonstrated that osteogenesis occurred in the cells cultured for 21 days in the osteogenic-promoting medium.

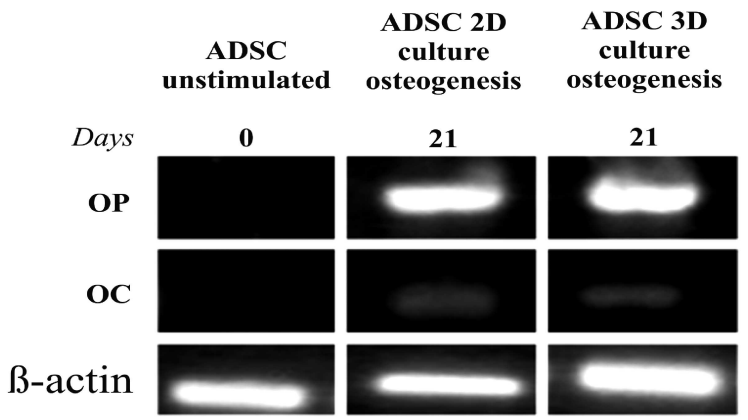

Fig. (5). Detection of osteogenesis-specific genes. To confirm osteogenesis after 21 days, the expression of osteopontin (lane A) and osteocalcin (lane B) was examined with RT-PCR in unstimulated ADSCs (channel 1) and stimulated ADSCs (with osteogenic medium) in 2D (channel 2) and 3D culture (channel 3). $\beta$-actin was used as a positive control (lane C).
To apply this system toward cell-based, skeletal tissue engineering efforts, the 3D chitosan scaffold must be shown to be effective in calvarial defect regeneration. Subcutaneous implants of uncolonized 3D matrices or 3D matrices colonized with ADSCs resulted in a mild inflammatory infiltrate in the first weeks after implantation (Fig. 6a, d). In the weeks after reducing the intensity of the matrix fibers, this infiltrate migrated from the periphery to the center of the implant (Fig. 6a, b, c, e, f). The increase in inflammatory infiltrate may have occurred due to an increase in the new formation of blood vessels throughout the study period (shown by black arrows). This new vessel formation occurred in both groups after the third week following implantation (Fig. $\mathbf{6 b}, \mathbf{c}$ ), and the new vessel formation intensified in the fifth week (Fig. 6e, f). However, the number of new vessels was higher in the colonized 3D matrix, which may have been due to the ADSCs changing the environment (Fig. $\mathbf{6 g}, \mathbf{h}$ ). With increasing intensity of the inflammatory infiltrate, there was also an increase of reabsorptive cells after the third week following implantation. However, no inflammations were observed. The surrounding tissue was in its native

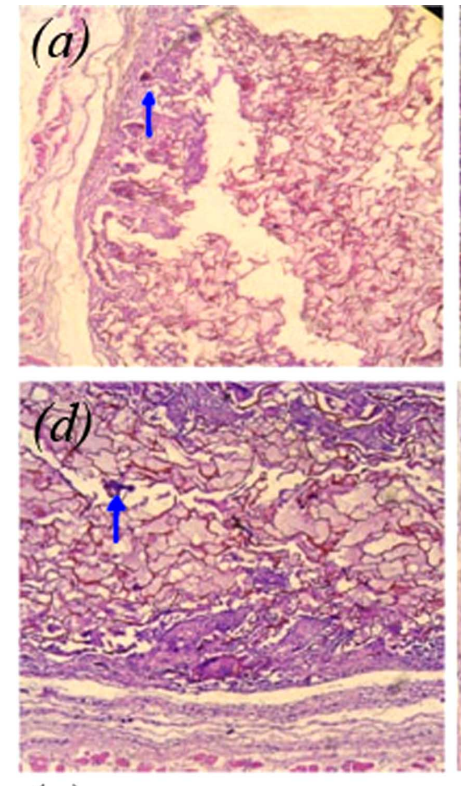

(g)

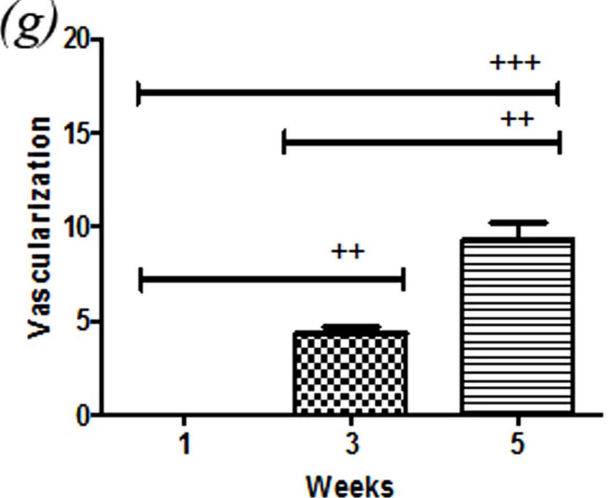

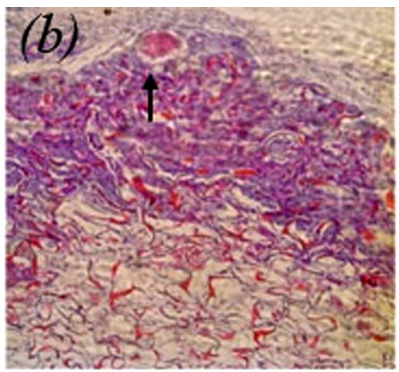
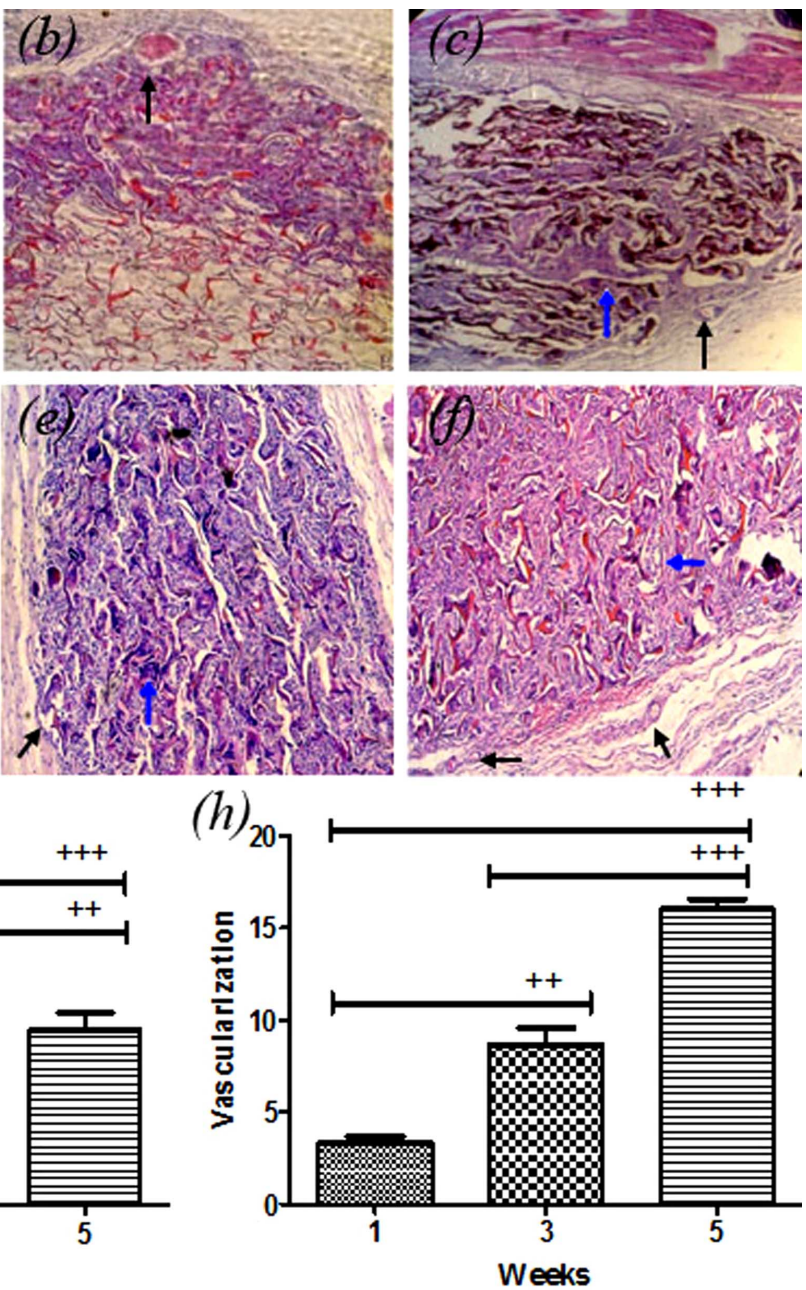

Fig. (6). Histological analysis of subcutaneous implants in vivo. Photomicrographs of histological sections of implants with uncolonized 3D matrices and 3D matrices colonized with ADSCs. Hematoxylin-eosin staining was used to compare sections. Histological sections corresponding to a) 1, b) 3 and c) 5 weeks after implantation of uncolonized 3D matrices are shown. Histological sections corresponding to d) 1 , e) 3 and f) 5 weeks after implantation of ADSCs-colonized 3D matrices are shown. Black arrows indicate neovascularization, and blue arrows indicate reabsorptive cells. Quantification of neovascularization after subcutaneous of implants of an $\mathbf{g}$ ) uncolonized 3D matrix and $\mathbf{h})$ a 3D matrix colonized with ADSCs. The symbols represent the following $p$-values: ${ }^{*} p<0.05$ compared between groups; and ${ }^{* * *} p<0.0001$ compared between groups. 
form, which suggested that the reabsorption process of the 3D matrix did not release toxic products that could damage the surrounding tissue. Furthermore, chitosan-based implants evoke a minimal foreign body reaction $[23,45,46]$.

Five weeks after the implantation in the calvarial defects, small bone repair implants were observed in both the uncolonized 3D matrices and the 3D matrices colonized by ADSCs. However, the level of bone maturation differed between these two groups. The newly formed bone tissue in the operation area had immature characteristics with many marrow spaces, and the newly deposited bone matrix in animals that received uncolonized 3D matrices had disordered osteocytes (Fig. 7a). Areas of immature bone tissue were observed in implants with ADSCs-colonized 3D matrices. In most samples, however, the histological appearance was organized with parallel lines of osteocytes and collagen lamellae, which indicated advanced bone maturation (Fig. 7b). Immunohistochemistry revealed the presence of EGFP ADSCs 5 weeks after implantation of the matrices colonized by ADSCs (Fig. 7c, d; shown by black arrows). Inflammation was not found in the calvarial defects containing the implants, but new bone formation was found in these calvarial defects. In the newly formed bone matrix, ADSCs labeled with EGFP were observed. Together, these data demonstrate the biocompatibility and effectiveness of chitosan-gelatin-chondroitin 3D matrices for use in bone tissue engineering.

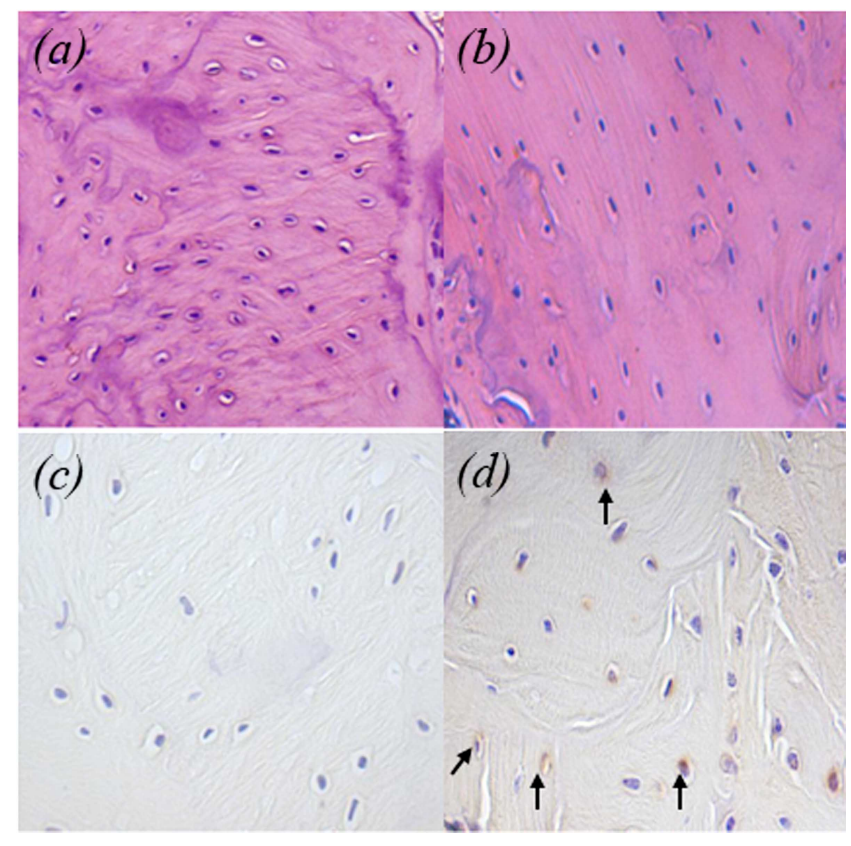

Fig. (7). Histological analysis of calvarial bone defect implants in vivo. a) Photomicrographs of histological sections of uncolonized 3D matrix implants after 30 days. Hematoxylin-eosin staining. Histological appearance of the healed, immature bone. b) Photomicrographs of histological sections of ADSCs-colonized 3D matrix implants after 30 days. Hematoxylin-eosin staining. Histological appearance of the healed bone showing lamellar characteristics and osteocytes lined in rows, which is typical of mature bone. c) Immunohistochemical EGFP analysis 30 days after implantation of uncolonized 3D matrices. c) Immunohistochemical EGFP analysis 30 days after implantation of ADSCs-colonized 3D matrices. Darkened reactions indicate the presence of EGFP.

\section{CONCLUSION}

Our in vitro results demonstrate the ability of chitosangelatin-chondroitin scaffold allow colonization and osteogenic differentiation of ADSCs. Furthermore in vivo results demonstrated that the chitosan-gelatin tridimensional scaffold promoted adequate healing and showed integration into the surrounding tissues, representing a potential carrier for the delivery of ADSCs in bone engineering development.

\section{CONFLICT OF INTEREST}

None declared.

\section{ACKNOWLEDGEMENTS}

The authors would like to acknowledge Conselho Nacional de Desenvolvimento Científico e Tecnológico $(\mathrm{CNPq} /$ Brazil), Fundação de Amparo à Pesquisa de Minas Gerais (FAPEMIG/Brazil) and Coordenação de Aperfeiçoamento de Pessoal de Nível Superior (CAPES) for supporting this work. The funders had no role in the study design, data collection, data analysis, decision to publish, or preparation of the manuscript. The authors also thank Juliana Lott Carvalho, Alessandra Arcoverde Cavalcanti Zonari, Pablo Herthel de Carvalho and Dawidson Assis Gomes for their intellectual support.

\section{REFERENCES}

[1] Porter JR, Timothy TR, Popat KC. Bone tissue engineering: A review in bone biomimetics and drug delivery strategies. Biotechnol Prog 2009; 25: 1549-60.

[2] Melton $\mathrm{LJ}_{3}{ }^{\text {rd }}$. Adverse outcomes of osteoporotic fractures in the general population. J Bone Miner Res 2003; 18: 1139-41.

[3] Johnell O, Kanis JA, Oden A, et al. Mortality after osteoporotic fractures. Osteoporos Int 2004; 15: 38-42.

[4] Nyman JS, Reyes M, Wang X. Effect of ultra structural changes on the toughness of bone. Micron 2005; 36: 566-82.

[5] Hou CH, Yang RS, Hou SM. Hospital based allogenic bone bank 10 years experience. J Hosp Infect 2005; 59: 41-5.

[6] Mankin H J, Hornicek FJ, Raskin K A. Infection in massive bone allografts. Clin Orthop Relat Res 2005; 43: 2 210-16.

[7] Burge R, Dawson-Hughes B, Solomon DH, Wong JB, King A Tosteson A. Incidence and economic burden of osteoporosisrelated fractures in the United States. J Bone Miner Res 2007; 22 465-75.

[8] Dawson-Hughes B, Looker AC, Tosteson AN, Johansson H, Kanis JA, Melton LJ $3^{\text {rd }}$. The potential impact of the National Osteoporosis Foundation guidance on treatment. Osteoporos Int 2011; 2: 41-52

[9] Gitelis S, Saiz P. What's new in orthopedic surgery. J Am Coll Surg 2002; 194: 788-91.

[10] Tsuchida H, Hashimoto J, Crawford E, Manske P, Lou J. Engineered allogeneic mesenchymal stem cells repair femoral segmental defect in rats. J Orthop Res 2003; 21: 44-53.

[11] Lee JH, Rhie JW, Oh DY, Ahn ST. Osteogenic differentiation of human adipose tissue-derived stromal cells (hascs) in a porous three-dimensional scaffold. Biochem Biophys Res Commun 2008 370: 456-60.

[12] Zuk PA, Zhu M, Mizuno H, et al. Multilineage cells from human adipose tissue: Implications for cell based therapies. Tissue Eng 2001; 7: 211-28.

[13] Zhu Y, Liu T, Song K, Fan X, Ma X, Cui Z. Adipose-derived stem cell: A better stem cell than bmsc. Cell Biochem Funct 2008; 26: 664-75.

[14] Witkowska-Zimny M, Walenko K. Stem cells from adipose tissue. Cell Mol Biol Lett 2001; 16: 236-57.

[15] Ge Z, Jin Z, Cao T. Manufacture of degradable polymeric scaffolds for bone regeneration. Biomed Mater 2008; 3: 1-11. 
[16] Costa-Pinto AR, Correlo VM, Sol PC, et al. Osteogenic differentiation of human bone marrow mesenchymal stem cells seeded on melt based chitosan scaffold for bone tissue engineering applications. Biomacromolecules 2009; 10: 2067-73.

[17] Puppi D, Chiellini F, Piras AM, Chiellini E. Polymeric Materials for bone and cartilage repair. Prog Polym Sci 2010; 35: 403-40.

[18] Khor E, Lim LY. Implantable applications of chitin and chitosan. Biomaterials 2003; 24: 2339-49.

[19] Di Martino A, Sittinger M, Risbud MV. Chitosan: a versatile biopolymer for orthopaedic tissue-engineering. Biomaterials 2005; 26: 5983-90.

[20] Huang Y, Onyeri S, Siewe M, Moshfeghian A, Madihally SV. In vitro characterization of chitosan-gelatin scaffolds for tissue engineering. Biomaterials 2005; 26: 7616-27.

[21] Machado CB, Ventura JMG, Lemos AF, Ferreira JMF, Leite MF, Goes AM. 3D chitosan-gelatin-chondroitin porous scaffold improves osteogenic differentiation of mesenchymal stem cells. Biomed Mater 2007; 2: 124-31.

[22] Howard D, Buttery LD, Shakesheff KM, Roberts SJ. Tissue engineering: strategies, stem cells and scaffolds. J Anat 2008; 213: 66-72.

[23] Miranda SCCC, Silva GAB, Hell RCR, Martins MD, Alves JB, Goes AM. Three-dimensional culture of rat BMMSCs in a porous chitosan-gelatin scaffold: A promising association for bone tissue engineering in oral reconstruction. Arch Oral Biol 2011; 56: 1-15.

[24] Mao JS, Zhao LG, Yin YJ, Yao KD. Structure and properties of bilayer chitosan-gelatin scaffolds. Biomaterials 2003; 24: 1067-74.

[25] Zou D, Zhang Z, He J, et al. Repairing critical-sized calvarial defects with BMSCs modified by a constitutively active form of hypoxia-inducible factor-1a and a phosphate cement scaffold. Biomaterials 2011; 32: 9707-18

[26] Breyner NM, Hell CR, Carvalho LRP, et al. Effect of a threedimensional chitosan porous scaffold on the differentiation of mesenchymal stem cells into chondrocytes. Cells Tissues Organs 2009; 190: 1-10

[27] Castanheira P, Torquetti LT, Magalhães DRS, Nehemy MB, Goes AM. DAPI Diffusion after intravitreal injection of mesenchymal stem cells in the injured retina of rats cell. Transplantation 2009; 18: 423-31.

[28] Otto WR, Rao J. Tomorrow's skeleton staff: mesenchymal stem cells and the repair of bone and cartilage. Cell Prolif 2004; 37: 97110 .

[29] Giusta MS, Andrade H, Santos AV, et al. Proteomic analysis of human mesenchymal stromal cells derived from adipose tissue undergoing osteoblast differentiation. Cytotherapy 2010;12: 47890.

[30] Ducy P, Schincke T, Karsenty G. The osteoblast: A sophisticated fibroblast under central surveillance. Science 2000; 289: 1501-4.
[31] Long MW. Osteogenesis and Bone-Marrow-Derived Cells. Blood Cells Mol Dis 2001; 27: 677-90.

[32] Hattori H, Sato M, Masuoka K, et al. Osteogenic potencial of human adipose tissue-derived stromal cells as an alternative stem cell source. Cells Tissue Organs 2004; 178: 2-12.

[33] Ogawa R, Mizuno H, Watanabe A, Migita M, Shimada T, Hyakusokua $\mathrm{H}$. Osteogenic and chondrogenic differentiation by adipose-derived stem cells harvested from GFP transgenic mice. Biochem Biophys Res Commun 2004; 313: 871-7.

[34] Vaux DL, Korsmeyer SJ. Cell death in development. Cell 1999; 96: 245-54.

[35] Rocha LB, Goissis G, Rossi MA. Biocompatibility of anionic collagen matrix as scaffold for bone healing. Biomaterials 2002; 23: 449-56.

[36] Lawrence BJ, Madihally SV. Cell colonization in degradable 3D porous matrices. Cell Adh Migr 2008; 2: 9-16.

[37] Thein-Han WW, Saikhun J, Pholpramoo C, Misra RD, Kitiyanant Y. Chitosan-gelatin scaffolds for tissue engineering: physicochemical properties and biological response of buffalo embryonic stem cells and transfectant of GFP-buffalo embryonic stem cells Acta Biomater 2009; 5: 3453-66.

[38] Meier P, Finch A, Evan G. Apoptosis in development. Nature 2000; 407: 796-80.

[39] Jaiswal N, Haneynesworth SE, Caplan AI, Bruder SP. Osteogenic differentiation of purified, culture expanded human mesenchymal stem cells in vitro. J Cell Biochem 1997; 64: 295-312.

[40] Christenson RH. Biochemical markers of bone metabolism: an overview. Clin Biochem 1997; 30: 573-93.

[41] Medrado GCB, Machado CB, Valério P, Sanches MD, Goes AM. The effect of a chitosan-gelatin matrix and dexamethasone on the behavior of rabbit mesenchymal stem cells. Biomed Mater 2006; 1 : 155-61.

[42] Huang JI, Beanes SR, Zhu M, Lorenz P, Hedrick MH, Benhaim P. Rat extramedullary adipose tissue as a source of osteochondrogenic progenitor cells. Plast Resconstr Surg 2002; 109: 1033-41.

[43] Liu F, Malaval L, Aubin JE. Global amplification polymerase chain reaction reveals novel transitional stages during osteoprogenitor differentiation. J Cell Sci 2003; 116: 1787-96.

[44] Klein-Nulend J, Roelofsen J, Semeins CM, Bronckers ALJJ, Elisabeth H, Burger EH. Mechanical stimulation of osteopontin mRNA expression and synthesis in bone cell cultures. J Cell Physiol 1997; 170: 174-81.

[45] Muzzarelli RA, Mattioli-Belmonte M, Tietz C, et al. Stimulatory effect on boneformation exerted by a modified chitosan. Biomaterials 1994; 15: 1075-81.

[46] Klokkevold PR, Vandemark L, Kenney EB, Bernard GW. Osteogenesis enhanced by chitosan (poly-Nacetylglucosaminoglycan) in vitro. J Periodontol 1996; 67: 1170-5.

(C) Carvalho et al.; Licensee Bentham Open.

This is an open access article licensed under the terms of the Creative Commons Attribution Non-Commercial License (http://creativecommons.org/licenses/by$\mathrm{nc} / 3.0 /$ ), which permits unrestricted, non-commercial use, distribution and reproduction in any medium, provided the work is properly cited. 